\title{
L'élaboration de la politique de décentralisation au Mali : des logiques plurielles
}

\section{Julien Félix}

\section{OpenEdition}

\section{Journals}

Édition électronique

URL : http://journals.openedition.org/apad/861

DOI : 10.4000/apad.861

ISSN : 1950-6929

Éditeur

LIT Verlag

Édition imprimée

Date de publication : 1 mai 1996

Référence électronique

Julien Félix, «L'élaboration de la politique de décentralisation au Mali : des logiques plurielles », Bulletin de l'APAD [En ligne], 11 | 1996, mis en ligne le 03 juillet 2007, consulté le 07 septembre 2020. URL : http://journals.openedition.org/apad/861 ; DOl : https://doi.org/10.4000/apad.861

Ce document a été généré automatiquement le 7 septembre 2020

Bulletin de l'APAD 


\title{
L'élaboration de la politique de décentralisation au Mali : des logiques plurielles
}

\author{
Julien Félix
}

1 Les champs politique et social maliens ont été bouleversés ces dernières années: renversement du régime de Moussa Traoré, intervention de la Banque Mondiale et du Fonds Monétaire International, séparatisme touareg, troubles sociaux permanents dans les villes comme dans les campagnes, chute du prix du coton, etc. A ces ruptures et à la désagrégation de l'Etat qu'elles ont entraînée, répondent des processus de reconstruction: développement des organisations non gouvernementales (ONG) locales, multiplication des organisations paysannes, introduction du multipartisme, renouvellement de la classe politique. L'ensemble de ces transformations a conduit à la modification des modalités de fonctionnement de l'Etat et, entre autres, a orienté les décideurs vers une décentralisation des institutions publiques. Cette décentralisation a pour principal objectif la constitution de collectivités locales aux pouvoirs réels (selon le principe de subsidiarité ${ }^{1}$ ) dotés d'une personnalité juridique, d'un pouvoir réglementaire et d'une autonomie financière. La mise en place d'une telle politique procède d'une rupture manifeste avec le système politico-administratif né des indépendances et, à ce titre, justifie notre intérêt.

2 Mais s'il y a rupture, il n'y a pas nouveauté. En effet,. force est de constater que l'idée d'une décentralisation des structures politico-administratives maliennes n'est pas originale. Dès 1979, une réforme institutionnalise des assemblées élues - les conseils locaux - ayant pour rôle la surveillance de la structure administrative déconcentrée responsable de la formulation de programmes de développement régionaux - le comité local de développement. Il ne manque à ce dispositif, pour pouvoir être considéré comme un acte de décentralisation des structures politico-administratives, que des moyens financiers propres. Cette seconde étape est franchie en 1988 avec la création du "fonds de développement local et régional", institution chargée de financer les programmes des cercles et des régions. Mais ce fonds, malgré les dires des responsables 
politiques, ne sera jamais contrôlé par les collectivités locales. Les textes définissant ses règles de fonctionnement ne seront jamais promulgués et les autorités centrales l'utiliseront en fonction de leurs propres objectifs (par exemple, plus de $50 \%$ du fonds a été "mis en priorité" sur instruction présidentielle pour des usages discrétionnaires (Hall, Magassa, Ba, Hodson, 1991 : 18).

3 La politique de décentralisation introduite pendant cette période reste donc largement inachevée. Cette situation est le résultat d'une série de blocages institutionnels dû à des conflits internes à l'élite politico-administrative. En effet, dès son introduction, la politique de décentralisation devient un enjeu central des relations de pouvoir entre le parti unique -la structure politique - et les membres de l'administration. Le système de décision est alors entièrement contrôlé par la haute administration, les membres des instances supérieures du parti sont marginalisés. Ces derniers veulent donc, afin de reconquérir un pouvoir perdu, multiplier les instances décisionnelles et renforcer le pouvoir des régions dans lesquelles ils ont une assise réelle. La politique de décentralisation est l'outil choisi par la classe politique pour reconquérir son pouvoir, ses avancées et ses reculades illustrent les rapports de force au sein de l'appareil étatique.

4 Aujourd'hui, la mise en place au Mali d'une politique de décentralisation semble faire l'unanimité des différents acteurs intervenant dans le cadre des "politiques globales" 2. Chacun de ces acteurs semble être convaincu par la nécessité d'une décentralisation et en demande, dans la mesure de ses moyens, l'application dans les plus brefs délais. Cette célérité est étonnante car, si l'on parle beaucoup de décentralisation en Afrique de l'Ouest, il est rare que pareil projet aboutisse. De plus, l'unité même des points de vue sur la décentralisation est inattendue. En effet, une politique publique de pareille ampleur est révélatrice d'un projet de société - caractéristique d'une idéologie - donc devrait être un enjeu sur lequel se cristallisent les positions ambivalentes des différents groupes sociaux ou, plus largement, des acteurs concernés. Or, dans le cas qui nous intéresse aucun débat, aucun affrontement, n'ont jamais eu lieu. Tous les acteurs ayant voix au chapitre dans la définition des politiques globales au Mali partageraient-ils un projet de société identique?

5 Posée en ces termes, la question de départ proposée offre une hypothèse claire à ce travail. En effet, nous nous proposons de démontrer que si la politique de décentralisation fait l'unanimité, cette unanimité ne peut que cacher des projets de société différents (pour ne pas dire opposés).

6 Autrement dit, si les textes - consensus minimum à toute action - sont les mêmes pour chacun des acteurs, les modalités d'application imaginées -la politique n'est pas encore entrée en vigueur-répondent à des logiques diverses. Avant d'entrer dans le vif du sujet et de proposer l'image que chaque acteur se fait de la décentralisation et l'utilité de cette dernière quant à la réalisation de son projet de société, énumérons rapidement ceux sur lesquels nous nous concentrons. Il s'agit du gouvernement - ou plus largement de la nouvelle classe politique malienne -, de deux des institutions de Bretton Woods la Banque Mondiale et le Fonds Monétaire International - et des grandes organisations d'aide au développement ${ }^{3}$.

7 Le premier acteur dont nous allons parler est l'actuel gouvernement malien ou, plus largement, une grande partie de la nouvelle classe politique. Il organise la décentralisation pour atteindre les objectifs politiques que sont la légitimation de l'Etat de droit et la participation populaire aux processus de prise de décision. La Banque 
Mondiale et le Fonds Monétaire International constituent le second des acteurs analysé. Le Mali étant soumis à un plan d'ajustement structurel, ces deux institutions prennent activement part aux décisions concernant la politique économique et financière mais aussi à l'ensemble des choix effectués par le gouvernement malien.

8 La décentralisation est à leurs yeux un moyen, parmi d'autres, permettant la diminution du déficit budgétaire de l'Etat. Le troisième acteur est composé des coopérations bilatérales (France, Suisse, etc.), multilatérales (CILSS, Club du Sahel, etc.) et de certaines grandes ONG (OXFAM, etc.). Il a un poids financier réel et une influence non négligeable. Il est aussi intéressé par la politique de décentralisation mais dans le cadre d'une politique de "développement local".

9 Le fait que ces trois acteurs prônent une politique de décentralisation explique la rapidité des réformes envisagées et l'absence de blocage. Mais, comme proposé ci-dessus, sa rapide planification n'est pas, à notre avis, le résultat d'un consensus mais une juxtaposition de lectures différentes offrant à chacun l'illusion d'un accord sur l'essentiel. En fait, chacun voit dans la politique de décentralisation une réponse aux problèmes qu'il juge fondamentaux et ignore les autres discours, les croyant complémentaires ou espérant réussir à imposer le sien lors de sa mise en place effective de la politique.

10 Cet article est organisé en trois chapitres présentant chacun un acteur et sa lecture de la politique de décentralisation. Chaque chapitre offre une mise en contexte de l'acteur, les objectifs auxquels répond la politique de décentralisation qu'il veut mener et les problèmes auxquels il se trouve confronté. La conclusion essaie de définir, à l'aide du révélateur qu'est la politique de décentralisation, les projets de société de chacun des acteurs $^{4}$.

Pour la nouvelle classe politique malienne : un outil au service de la démocratie

11 En 1991, des manifestations de grandes ampleurs mettent le régime autocratique de Moussa Traoré dans une situation politique précaire. Ces manifestations sont durement réprimées par l'armée qui, devant l'impasse de la répression, finit par se décider à renverser le président. Après une phase de transition pendant laquelle sont créés de multiples partis politiques, des élections législatives, puis présidentielles, sont organisées. Durant cette période une "société civile" ${ }^{5}$ se constitue : les coordinations d'étudiants, de lycéens, de fonctionnaires, d'ouvriers, de commerçants, se multiplient et revendiquent chacune les fruits de la démocratisation. Par ailleurs, les organisations de développement rural (ONG, associations villageoises, etc.) mettent à profit la libéralisation politique pour, d'une part, réclamer une plus large autonomie - elles prennent leurs distances par rapport aux politiques étatiques et construisent leurs propres programmes de développement - et, d'autre part, s'interroger sur le bien fondé de la politique de développement rural proposée par le gouvernement et/ou les sociétés d'Etat ayant à charge l'organisation des opérations de développement.

12 L'expression sans cohérence de ces intérêts corporatistes et/ou régionaux, pas toujours représentatifs des groupes sociaux composant la société malienne, met en péril l'existence du nouvel Etat de droit qui émerge après 1991 (ce d'autant plus que les manifestations auxquels elle donne lieu sont souvent violentes). Paradoxalement, les nouveaux partis politiques, organisés sur des bases idéologiques ou clientélistes, ne peuvent jouer le rôle de médiateur entre la société civile et le pouvoir. Ils sont, dès leur apparition, considérés par les Maliens comme illégitimes car répondant à leurs seuls intérêts opportunistes. 

étroitement liés : le premier est la déliquescence de l'Etat, le second l'élaboration d'une politique générale cohérente répondant aux nouvelles demandes sociales et le troisième la mise en place de cette politique.

une nouvelle légitimité à l'Etat et élaborer une politique générale cohérente demande la participation des citoyens aux processus de décisions qui les concernent Cette obligation requiert des institutions dont la fonction est d'être des intermédiaires entre la société civile et le pouvoir d'Etat. Cette fonction n'étant pas remplie par les partis politiques, la seule solution qui se propose au gouvernement malien est de constituer de nouveaux "espaces de médiation". Un espace de médiation est, dans notre cas d'étude, une institution permettant la construction - par un système politique consensuel ou majoritaire - de politiques globales et/ou particulières reconnues par l'ensemble des acteurs concernés. Ces nouveaux espaces de médiation doivent, afin d'être occupés par les citoyens, être proches de ces derniers, traiter de leurs préoccupations concrètes et posséder un véritable pouvoir de décision. Ils doivent concerner la totalité du territoire et tous les niveaux de son organisation.

Dans cette optique, la politique de décentralisation, en raison de la multiplication des institutions politiques qu'elle permet et de leur répartition équilibrée sur l'ensemble du territoire, est une solution appropriée aux problèmes posés. Tout d'abord, elle permet la participation des citoyens, de tous les citoyens, au processus décisionnel et par là même, leur responsabilisation. Elle rend lisible les enjeux du développement et offre une place à l'Etat dans le processus de la démocratisation, donc en reconnaît le rôle et en légitime l'existence. Ensuite, cette politique permet d'organiser la demande sociale donc l'élaboration de politiques globales et/ou sectorielles reconnues par l'ensemble des acteurs concernés. Enfin, la proximité entre l'Etat et ses administrés permet une application plus rapide et plus efficace des décisions prises.

La politique de décentralisation offre donc à l'Etat une légitimation nouvelle et un outil efficace quant à l'élaboration des politiques publiques et à leur exécution. Elle permet la constitution d'un Etat de droit, renforce les processus de démocratisation et, à ce titre, contente l'ensemble de la classe politique.

Par ailleurs, la politique de décentralisation offre à la nouvelle classe politique deux autres avantages non négligeables. Le premier est la mobilisation de l'opinion publique autour d'un sujet dont elle est un des acteurs privilégiés. Après avoir été considérée comme nuisible, la classe politique retrouve, en partie, la valorisation sociale inhérente à son statut d'élite. La mise en place de la politique de décentralisation ne restaure donc pas uniquement la légitimité de l'Etat, mais aussi celle de ses promoteurs. Ce processus n'est pas le fait du hasard, au contraire, il a bénéficié d'une mise en scène particulière dont la pièce maîtresse est l'absence totale de référence à la politique de déconcentration-décentralisation conduite sous l'ancien régime. Cet oubli montre clairement l'intention de la classe politique de se démarquer de tout passé ou, plus précisément, sa volonté de s'approprier totalement une innovation porteuse d'espoir. La décentralisation n'a pas été élaborée pour réaménager des institutions mises en place par l'ancien parti unique. mais pour donner vie à une nouvelle utopie et créer un projet mobilisateur au service d'une nouvelle classe politique ${ }^{6}$.

18 Le second avantage, pour le gouvernement et la nouvelle classe politique, présenté par la politique de décentralisation est une plus grande marge de manœuvre en ce qui 
concerne la négociation sur le statut du Nord du Mali. En effet, la décentralisation permet au gouvernement de discuter sur une possible autonomie de cette région sans perdre la face ou être accusé de vendre la Nation. Elle dépassionne la discussion sur les revendications touarègues en l'immergeant dans un débat national dont les enjeux sont compris de tous.

Toutefois, comme nous l'avons dit, donner une nouvelle légitimité à l'Etat et élaborer une politique générale cohérente demande la participation des Maliens aux espaces de médiation construits par la politique de décentralisation. Or, cette participation nécessite des conditions générales dont on peut douter qu'elles soient actuellement toutes réunies. La principale de ces conditions est l'existence d'une société civile sur l'ensemble du territoire. En effet, pour participer au jeu politique il faut posséder une conscience politique, être libre de ses choix et appartenir à des organisations qui permettent la construction d'un projet. Ce sont ces organisations, intégrées, qui constituent la société civile.

Dans cette optique, le problème concret qui se pose au Mali est la disparité de la densité d'organisations à participation volontaire (ou communautés d'adhésion), notamment dans le monde rural. Aussi, dans certaines régions, le risque est grand de voir les institutions construites par la politique de décentralisation n'être que des coquilles vides ou les chambres d'enregistrement de décisions prises par les autorités traditionnelles. Ce cas risque d'être particulièrement fréquent car la commune, association de plusieurs villages et espace de médiation le plus décentralisé, possède toutes les caractéristiques permettant son appropriation par les lignages dominants ou une reproduction des rapports de pouvoir construit par la tradition ${ }^{7}$.

Par ailleurs, force est de constater que même dans les régions ou les organisations à participation volontaire sont nombreuses, il n'est pas certains que les Maliens investissent les espaces de médiation mis à leur disposition. En effet, la participation à des structures politiques demande aux villageois l'abandon de leur stratégie d'observation passive ou le dépassement de facteurs contraignants (par exemple un système de production articulé au marché ${ }^{8}$ ). Dans tous les cas, cette participation sera déterminée par la logique de la minimisation des risques propre aux sociétés rurales.

En résumé, l'objectif du gouvernement et plus largement de la nouvelle classe politique malienne est l'établissement d'un Etat de droit légitime permettant l'organisation de la demande sociale ou, plus largement, la construction d'un système politique démocratique. Dans ce cadre, la politique de décentralisation permet la constitution d'espaces de médiation au sein duquel les acteurs de la société civile peuvent définir des politiques de développent acceptée de tous. Elle est donc un instrument particulièrement approprié à la problématique actuelle, mais peut se trouver fragilisée par la disparité des densités des communautés d'adhésion - base de la société civile - et la prégnance des rapports de pouvoir issus de la tradition et/ou de la logique de minimisation des risques.

Pour les institutions de Bretton Woods : un outil au service du marché

Le second acteur ayant un rôle important dans la définition des politiques globales au Mali est l'ensemble formé par deux des institutions de Bretton Woods, soit la Banque Mondiale et le Fonds Monétaire International. Ces deux institutions interviennent au Mali à la faveur d'un plan d'ajustement structurel dont les objectifs sont la réduction des déficits budgétaires de l'Etat et le rétablissement des grands équilibres macro-économiques (balance des paiements, inflation, ...). Pour atteindre ces derniers, 
la Banque Mondiale et le Fonds Monétaire International contrôlent étroitement le budget de l'Etat et donnent une nouvelle orientation à certaines de ses politiques; direction qui, en règle générale, va dans le sens d'une libéralisation de la sphère économique et a pour conséquence la redéfinition du rôle de l'Etat. En effet, ce dernier n'a plus à être le moteur du développement - rôle qui lui incombait dans le passé -, mais doit se contenter de mettre en place les modalités qui permettent d'accroître le rôle du secteur privé. Cette transformation substantielle a des conséquences directes sur son fonctionnement et sur son organisation. D'une part, il est tenu de diminuer son coût de fonctionnement (réduction des déficits budgétaires) et, d'autre part, il doit réduire son champ d'intervention (libéralisation de l'économie).

Dans ce cadre, une politique de décentralisation est vivement encouragée par les institutions de Bretton Woods, car elle doit permettre à l'Etat un allégement de ses structures et une meilleure utilisation des moyens à disposition, donc la diminution de ses coûts de fonctionnement. Ces objectifs sont indéniablement utiles; mais ces institutions semblent oublier les investissements inhérents à la mise en place de cette politique. Or, dans le cas du Mali, la constitution de l'ensemble des institutions décentralisées demandera des moyens humains, matériels et financiers importants. Des moyens humains et matériels car la mise sur pied de pouvoirs locaux capables d'assumer les tâches de développement ne peut se faire sans la formation des cadres, l'information et la formation des élus et des citoyens et la mise à disposition d'un ensemble de moyens matériels aux nouvelles institutions. Des moyens financiers car la formation des différentes institutions décentralisées demandera la création de budgets locaux (au niveau des régions, cercles et communes) financés, partiellement tout au moins, par l'Etat En effet, les recettes apportées par les taxes et impôts locaux - même si le taux de recouvrement de ces dernières se trouve être particulièrement fort seront beaucoup trop faibles pour constituer des budgets capables de financer des projets de développement et, par là-même, de mobiliser les populations. La démonstration de l'efficacité de la décentralisation nécessitera donc une contribution financière de l'Etat aux budgets des institutions décentralisées ou, à défaut de cette dernière, un recours aux bailleurs de fonds internationaux. Ainsi, la politique de décentralisation risque, non seulement de ne pas permettre la diminution des prestations financières de l'Etat central, mais au contraire d'accroître ses dépenses.

En fait, la décentralisation dans un pays comme le Mali, ou la plupart des besoins de la population, en terme de services publics, ne sont pas couverts, multiplie les arènes administratives et politiques sans en éliminer. Elle est une politique d'autant plus coûteuse que la démultiplication des instances de décision va générer des revendications particulières et/ou clientèlistes: les politiciens, dépendant de la réalisation de leurs objectifs pour assurer leurs réélections, vont faire pression sur le gouvernement afin de se voir attribuer effectivement subventions et crédits ${ }^{9}$.

En regard des objectifs de la Banque Mondiale et du Fonds Monétaire International, il est difficile de penser qu'ils souscrivent à une définition de la décentralisation autorisant une augmentation des dépenses de l'Etat malien. $\mathrm{n}$ est probable que pour les institutions de Bretton Woods, l'Etat central doit limiter ses prestations au maximum et ne pas fournir de subventions aux gouvernements locaux, sinon dans le strict cadre d'un équilibre budgétaire. Ces derniers devront alors assumer les charges du développement avec leurs propres ressources. Or, il est évident que ces gouvernements locaux n'auront pas les moyens matériels et financiers permettant le maintien des 
actuels - et déficients - services publics, ni donc, à plus forte raison ceux d'en permettre la création de nouveaux. Ils se verront donc rapidement dans l'obligation de faire recours au service de privés ou d'organiser les services mis à disposition des populations selon les lois du marché (la production et l'entretien des services publics seront alors à la charge des usagers).

Ainsi, il est probable que la politique de décentralisation, dans l'idéal des institutions de Bretton Woods, a pour objectif principal l'introduction d'une logique de marché dans le fonctionnement des institutions publiques et/ou une délégation des responsabilités étatiques aux organisations privées. Cette dernière remarque est confirmée par le fait que la Banque Mondiale et le Fonds Monétaire International parlent peu, dans le cadre de la politique de décentralisation, de l'Etat et de son rôle, mais mettent l'accent sur l'organisation de la société civile au niveau local. En effet, ces deux institutions encouragent la constitution d'organisations locales pouvant, à terme, fournir des prestations d'intérêt public. La question qui se pose est alors de savoir si le local ne devient pas une fin en soi et si la politique de décentralisation n'est pas la mise en forme de l'idée de marché ${ }^{10}$.

En résumé, l'objectif de la Banque Mondiale et le Fonds Monétaire International dépasse très largement le rétablissement des équilibres macro-économiques et budgétaires. L'intérêt que ces deux institutions portent à la politique de décentralisation et à la constitution d'organisations locales en témoigne. En fait, la réduction des déséquilibres est prétexte à une intervention qui a pour finalité l'introduction d'une logique de marché embrassant l'ensemble de la société malienne. Suivant ce raisonnement, les institutions de Bretton Woods ont tout intérêt à un désengagement de l'Etat au bénéfice du secteur privé, désengagement dont l'outil est la politique de décentralisation ${ }^{11}$.

Pour les instances de coopération, un outil au service du développement local

Le Sahel est une région particulièrement choyée par les différentes organisations d'aide au développement. Mais, l'apport d'une aide ne se fait pas sans contrepartie, entre autres en terme de partage du pouvoir politique. Ceci explique le fait que les donateurs occupent une position de premier ordre dans les prise de décisions concernant les politiques globales au Mali. Certains d'entre eux ont mis à profit cette position pour encourager la politique de décentralisation. En effet, plusieurs des organisations d'aide au développement - et non des moindres - font actuellement du "développement local" leur discours favori. Ces termes recouvrent un ensemble de réalités et de fantasmes. Dans le cadre dont nous traitons ici, ce discours peut être défini par la volonté de rapprocher le citoyen des institutions qui régissent sa vie de tous les jours. Son objectif premier est donc une réelle participation des populations aux processus de décision et leur responsabilisation quant à la gestion de leurs problèmes; en règle générale cette approche trouve un ancrage au niveau villageois ou intervillageois.

Jusqu'à présent, faute d'un cadre juridique offrant aux villages ou aux regroupements villageois une autonomie réelle, les réalisations s'inspirant de ce discours étaient confinées à des expériences pilotes. Dans l'optique de leur généralisation, les différentes organisations d'aide au développement impliquées ont donc naturellement encouragé l'élaboration d'une politique de décentralisation offrant un cadre juridique approprié à leurs projets.

31 Regardons plus précisément quels sont les champs d'action du développement local et leurs relations avec la politique de décentralisation. Schématiquement, il est possible 
de discerner dans le discours du développement local deux problématiques différentes : la première concerne la gestion des ressources naturelles et le seconde la production de services publics.

Suite à des sécheresses successives, les intervenants extérieurs ont porté une attention toute particulière aux problèmes touchant à la gestion des ressources naturelles. La plupart des activités conduites dans ce domaine - souvent sous forme de recherche-action - ont abouti à la conclusion que le problème majeur, quant à la gestion de ces ressources, est le manque d'institutions légitimes capables de construire et d'imposer des règles à l'ensemble de leurs utilisateurs (la déstructuration du monde rural semble telle qu'il n'y a plus d'institutions locales traditionnelles aptes à gérer les ressources naturelles au nom de la collectivité). Ces observations ont donné naissance aux politiques de "gestion des terroirs"; politiques dont l'objectif est d'appuyer la constitution d'institutions locales nouvelles qui permettent la gestion efficace des ressources naturelles ${ }^{12}$. Dans ce cadre, la politique de décentralisation - ou plus particulièrement l'autonomie qu'elle laisse aux communautés locales - est un élément indispensable à la généralisation de l'expérience susmentionnée. En effet, comment concilier un Etat centralisateur, seul producteur de règles et de normes, avec des institutions villageoises autonomes ne reconnaissant que les leurs. La politique de gestion des terroirs demande, pour être généralisée, une liberté d'action du local reconnue par l'Etat central et l'affectation des moyens humains et financiers correspondants aux coûts entraînés par les responsabilités déléguées. Autant de requêtes auxquelles la politique de décentralisation donne forme.

Le second champ d'activité investi par la politique du développement local est la production de services publics. Face à la faible couverture et à la mauvaise qualité des services publics offerts par l'Etat, les donateurs voient dans les collectivités locales, parce qu'elles rapprochent - sinon confondent - les producteurs et les usagers de ces services, le lieu idéal quant à leur production et à leur gestion. Mais, en raison de la non-reconnaissance par l'Etat d'une institution villageoise dotée d'une personnalité juridique, les organisations d'aide au développement intervenant dans ce domaine ont été, jusqu'à présent, dans l'obligation de s'adresser directement à certaines communautés d'adhésion censées représenter les intérêts du village ${ }^{13}$. Conscients de ce problème, elles encouragent aujourd'hui la constitution d'institutions locales de droit public représentatives des intérêts de l'ensemble de la population et bénéficiant d'une autonomie dans le domaine de la production et de la gestion des services publics. Elles ne peuvent donc que soutenir la mise en place de la politique de décentralisation qui, par la constitution des communes, répond à leur demande.

Les avantages indiscutables pour le développement local d'une politique de décentralisation ayant été posés, regardons maintenant quelles peuvent être les ambiguités ou les contradictions entre ces deux formes d'intervention. Deux observations peuvent être faite : la première concerne les méthodes d'approche même des deux politiques qui nous intéressent. Elles semblent être contradictoires: logique descendante dans le cas de la politique de décentralisation et logique ascendante dans celui de la politique de développement local. Quant à la seconde observation, elle soulève les éternels problèmes posés par la présence de plusieurs législateurs : cohésion des politiques, conflits de compétences. mais ne s'identifient pas l'une à l'autre. En effet, le processus de construction d'une 
institution villageoise légitime, représentative et responsable - objectif principal de la politique du développement local - est un processus lent, qui demande un appui adapté au cas par cas. Il ne peut pas être imposé unilatéralement sur l'ensemble d'une région à plus forte raison d'un pays. Il peut donc bénéficier de la décentralisation, mais ne s'y identifie pas: la décentralisation est une délégation de pouvoir de la pan de l'Etat central aux pouvoirs locaux, le développement local est une reconstruction du pouvoir local par les acteurs locaux.

On peut même se demander si la décentralisation ne met pas, partiellement, en péril les expériences menées par la politique de développement local. En effet, la mise en place d'un cadre normatif unique peut, dans certains cas, déstabiliser les processus amorcés ou même en retarder, vu la possible méfiance des populations envers toute intervention étatique, l'émergence. Par ailleurs, la décentralisation ne reconnaissant pas l'identité villageoise (l'institution la plus décentralisée, la commune, regroupe plusieurs villages), elle limite largement l'efficacité de la politique de développement local pour laquelle le lieu d'action pertinent, car le plus apte à être reconnu comme unité légitime, est le village.

Ainsi, dans l'idéal des acteurs prônant le développement local, la décentralisation se doit d'offrir un cadre législatif souple à l'extrême. Cette opinion pose de manière non dissimulée le problème de la cohésion des politiques. Les populations, disposant d'une importante autonomie au niveau le plus décentralisé, sont-elles libres de tout réinventer, dans les domaines qui les intéressent, ou doivent-elles se plier aux schémas directeurs élaborés par des techniciens désireux d'atteindre une certaine cohérence dans l'offre de service proposé au niveau d'une région (JACOB, 1993: 4). A cette interrogation les donateurs ne répondent pas explicitement, mais le seul fait qu'ils donnent la priorité, dans leur discours, à l'autonomie des communautés villageoises (autonomie qui permet à ces dernières la construction de règles légitimes concernant la gestion des ressources naturelles et la production de services publics appropriés et gérés efficacement) et ne précisent nulle part la place de l'Etat central et des niveaux de décision intermédiaires permet de présumer de la réponse. La situation conduit alors naturellement à la multiplication des programmes, ce qui peut aboutir à la coexistence - dans une même région - de plusieurs politiques sectorielles - avec les problèmes de cohérence que cela peut poser - et/ou des conflits de compétence structurels (en cas d'incompatibilité entre les politiques des différentes communautés villageoises ou entre celles des communautés villageoises et celles des niveaux politico-administratif supérieurs). Ces conflits semblent, dans le cas malien, d'autant plus probables que les attributions des différents échelons politico-administratifs se recoupent très largement, notamment en ce qui concerne l'élaboration des programmes de développement, l'établissement des plans d'aménagement de l'espace et la protection de l'environnement. (Mission de décentralisation, 1993a : 7) ${ }^{14}$.

Ces deux observations, concernant les difficultés d'une relation harmonieuse entre la politique de décentralisation et le discours du développement local, ont le mérite de réintroduire l'Etat dans la discussion, sujet quelque peu oublié par les organisations d'aide au développement. L'Etat, les modalités touchant à son action et les éléments précisant son rôle, semble peu les préoccuper : il est perçu au mieux comme un élément de la société offrant des opportunités à leurs actions (la politique de décentralisation en est une), au pire comme un obstacle à leurs projets. 
39 En résumé, l'objectif des organisations d'aide au développement est la constitution d'institutions locales légitimes, capables de gérer les ressources naturelles efficacement et de

fournir des services publics correspondant aux besoins des populations. Dans ce cadre, la politique de décentralisation offre à ces institutions des bases légales bienvenues, mais procède d'une logique qui peut sembler contraignante aux yeux des donateurs.

Conclusion

41 La décentralisation, actuellement élaborée au Mali, est encouragée par les trois grands types d'acteurs qui définissent les politiques globales de ce pays. Si, a priori, il existe un consensus sur l'idée de décentraliser le système politico-administratif et sur les textes qui vont permettre l'application de cette politique, il est évident que chaque acteur l'investit d'objectifs différents, révélateurs de projets de société distincts pour ne pas dire inconciliables. Notre conclusion se propose naturellement d'examiner leurs projets de société. A cette fin, il sera mené une analyse succincte du rôle donné par ces trois acteurs aux éléments de la société malienne que sont l'Etat, les collectivités territoriales décentralisées (locales) et la société civile. En effet, il nous semble que la place réservée à ces sujets permet de cerner les projets de société imaginés par la nouvelle classe politique malienne, les institutions de Bretton Woods et les organisations d'aide au développement ${ }^{15}$.

La représentation de l'Etat est particulièrement révélatrice des projets de société sous-jacents. Alors que l'Etat est défini positivement - son rôle est déterminé - et se trouve au centre du processus de décentralisation proposé par la nouvelle classe politique malienne, la place qu'il occupe dans les deux autres approches de la décentralisation est marginale et ses fonctions déterminées négativement (ces deux approches précisent ce que l'Etat ne doit pas faire et non pas ce qu'il fait). Plus précisément, chaque acteur imagine une forme d'Etat particulière. La nouvelle classe politique réinvente l'Etat de droit : il a pour objectif une démocratisation de la société et l'égalité des citoyens. L'Etat est maître de la production de services publics et joue le rôle d'organisateur du processus de développement économique (en cela, il ressemble à l'Etat providence). Les institutions de Bretton Woods rêvent de l'Etat libéral : il se doit d'être le plus discret possible et ne trouve sa place que dans la mise à la disposition de la société civile du cadre indispensable à son expression. La place centrale du processus de développement est alors occupée par le marché et l'ensemble des besoins définit en terme d'offre et de demande. Quant aux organisations d'aide au développement, elles pensent à l'Etat incitateur (BUETSCHI, CATTACIN, 1993 : 152) qui pousse la société civile à s'organiser et lui propose un encadrement. Son rôle est donc celui d'un animateur.

Deuxième élément : la société civile. Celle-ci est centrale dans les projets proposés par les institutions de Bretton Woods et par les organisations d'aide au développement. Dans les deux sociétés idéales imaginées par ces acteurs, la société civile est l'initiatrice du processus de développement. Mais la similitude s'arrête là car, si la Banque Mondiale et le Fonds Monétaire International la considèrent comme regroupant une pluralité d'acteurs aux relations concurrentielles définies par les lois du marché, les donateurs lui attribuent la gestion collective des biens communs et publics. Ainsi, si sa formation est encouragée par les uns et les autres, les principes qui l'animent, ou les modalités déterminant ses rapports internes, divergent profondément. Ce désaccord peut encore être étayé par deux autres points : tout d'abord, si les acteurs de la société civile apparaissant spontanément avec la naissance du marché pour les institutions de 
Bretton Woods, ils sont, pour les organisations d'aide au développement le résultat d'un long processus. Ensuite, si la société civile est le moteur du développement pour le premier des acteurs, seule son organisation sous forme d'institutions locales - résultat d'un processus ascendant - permet l'émergence d'un réel dynamisme_pour le second. Pour ce qui est du troisième acteur, la nouvelle classe politique, la considération qu'il porte à la société civile est importante, mais est étroitement liée à son inscription dans le cadre institutionnel pré défini par l'Etat. Cette dernière se voit donc imposer une construction organisée selon une logique descendante ${ }^{16}$.

Les collectivités territoriales, enfin, jouent des rôles différents dans chacun de ces projets de société. La nouvelle classe politique voit en elles des espaces de médiation, donc des lieux d'expression et de négociation. Elles sont, dans cette optique, partie prenante du système politico-administratif et prolonge un Etat central garant des droits démocratiques et de l'égalité entre citoyens. Proche de cette première proposition, les organisations d'aide au développement la nuanceraient toutefois suivant deux axes. Le premier est l'attribution prioritaire aux institutions locales, en sus de celle d'organisation de la demande sociale, de la production des services publics et des règles de gestion des ressources naturelles. Et le second, un rapport à l'Etat non pas défini par des règles hiérarchiques mais par des relations de confiance, résultat d'une autonomie totale des institutions locales (rappelons que dans l'optique des donateurs ces dernières sont l'aboutissement d'une logique ascendante ou agrégative). Quant aux institutions de Bretton Woods, troisième acteur concerné, force est de constater qu'elles attribuent une place très marginale aux collectivités territoriales et qu'elles semblent les considérer comme le simple relais de l'Etat dont, rappelons-le, le rôle est la promulgation de règles permettant la généralisation des lois du marché. En fait, cette absence de rôle spécifique attribué aux institutions locales confirme le désengagement de l'Etat proposé par ce dernier acteur.

Annexe 1 : les acteurs, leurs objectifs, solutions et problèmes

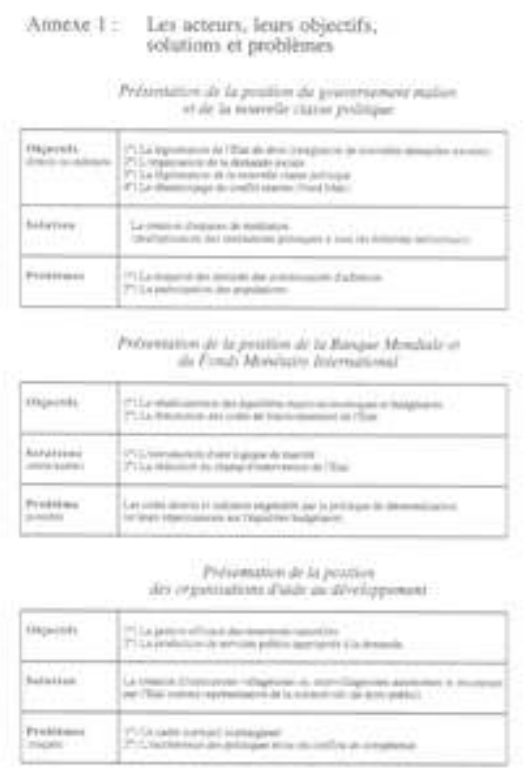

A voir en document annexe 
Annexe 2 : Les objectifs des acteurs et l'élaboration de la politique de décentralisation

Annexe 2: Les objectifs des acteurs et l'élaboration de la politique de décentralisation

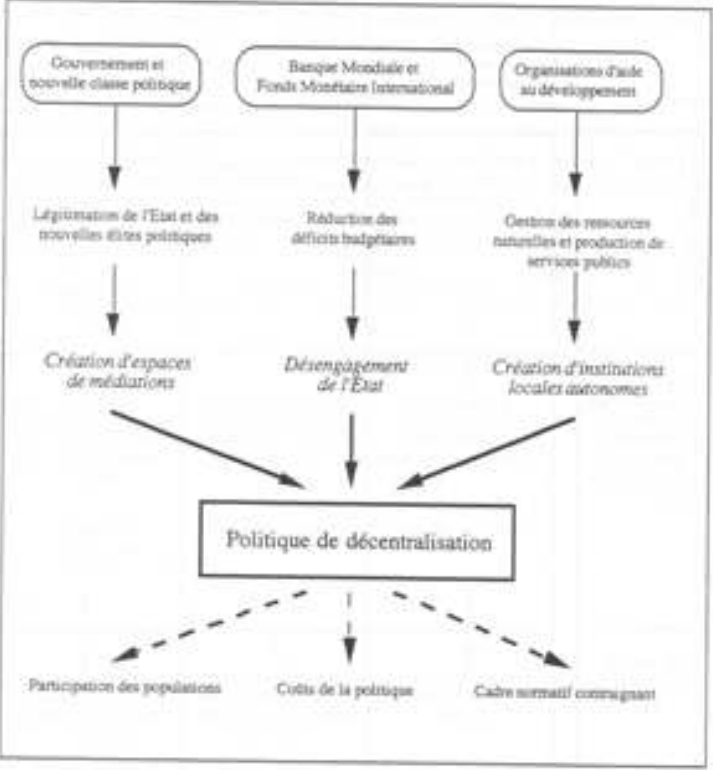

A voir en document annexe

Annexe 3 : Les projets de société des acteurs et la politique de décentralisation

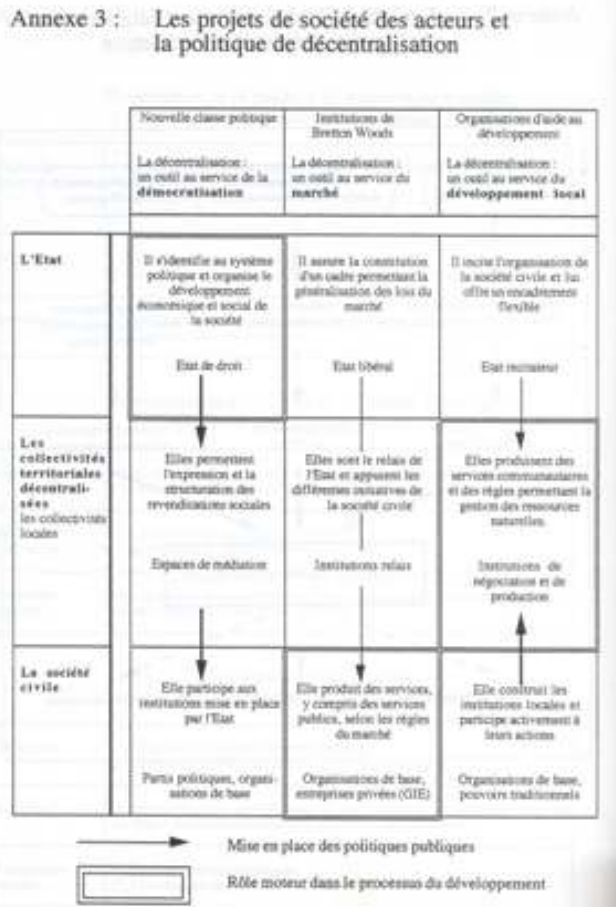

A voir en document annexe 


\section{BIBLIOGRAPHIE}

Lois et textes administratifs

Assemblée nationale

1993 Projet du code des collectivités territoriales

Mission de décentralisation

1994 Décentralisation, journal d'information et de réflexion sur la décentralisation

1993a Atelier national de concertation sur le projet de code des collectivités territoriales, rapport final, Bamako, Ministère de l'administration territoriale et de la décentralisation

1993b La décentralisation au Mali : stratégie générale de communication, Bamako, Ministère de l'administration territoriale et de la décentralisation

Présidence de la République 1993 Loi n 93008 déterminant les conditions de la libre administration des collectivités territoriales

Autres documents

Bertrand Monique, 1992 "Un an de transition politique : de la révolte à la troisième République" in : Politique Africaine ${ }^{\circ} 47$, pp. $9-22$

Buetschi Danielle, Cattacin Daniel, 1993 "L'Etat incitateur : nouvelles pratiques de la subsidiarité dans le système du bien-être suisse" in : Annuaire suisse de sciences politiques $n^{\circ} 33, \mathrm{pp}$. 143-162

Collectif, 1993 Décentralisation, organisations locales et ONG au Mali, Mémoire du séminaire de Bamako 29-31 mars 1993, Bamako, Centre Djoliba

1989 Les expériences d'une concertation, Rapport final de la rencontre régionale de Ségou sur la gestion des terroirs, Paris - Ouagadougou, Club du Sahel - CILSS

Chaumie J., 1985 "La gestion de l'environnement dans les pays sahéliens" in : Les cahiers de la recherche développement $n^{\circ} 8$, pp. 17-24

Chauvie Philippe, 1993 Commentaires sur le rapport final de l'atelier national de concertation sur le projet de code des collectivités territoriales, Vissoie, SEREC

Chauvie Philippe, Jacob Jean-Pierre, 1993 Mission d'appui à la décentralisation au Mali, Projet de Doussoudiana/Yanfolila, Vissoie - Genève, SEREC - IUED

Clauzel Jean, 1991 "L'évolution des structures de l'administration territoriale dans quelques Etats de l'Afrique francophone au sud du Sahara" in : Mondes et cultures, tome LI, $n^{\circ} 1-4$, pp.208-225

Conac Gérard, 1985 "Le processus de décision politique dans les Etats d'Afrique : exemples des politiques de l'eau" in : Les politiques de l'eau en Afrique, sous la direction de CONAC Gérard et Françoise, Paris, Economica.

DJjibo Hadiza, Coulibaly Cheibane, Marko Paul, Thomson James, 1991 Décentralisation, gouvernance et gestion des ressources naturelles renouvelables :options locales dans la République du Mali, Club du Sahel - CILSS - Associates in , Rural Development 
Faure Armelle, 1992 Perception de l'approche gestion des terroirs par les populations rurales, Ouagadougou, Ministère de l'agriculture et de l'élevage, Programme national de gestion des terroirs.

Hall Robert, Magassa Hamidou, Ba Aliou et Hodson Jeremy, 1991 L'organisation des services publics, la participation populaire et la décentralisation : options locales pour l'organisation et la production des services publics dans la République du Mali, Club du Sahel- CILSS - Associates in Rural Development

Helvetas

1994 Document de projet (version provisoire) :Programme développement rural et décentralisation, Zürich

Jacob Jean-Pierre, 1991 "Entre décentralisation et désengagement Principes et problèmes de l'auto-organisation paysanne en Afrique" in : Jeux et enjeux de l'auto-promotion, sous la direction de Kwan Kaï Hong, Genève, Cahiers de l'IUED, pp. 41-48

1993 Décentralisation : analyse écologique et gestion territoriale en Afrique tropicale sèche, manuscrit, Lausanne, UNIL

Programme national de gestion des terroirs, CILSS, 1993 Atelier national sur la problématique foncière et la décentralisation, document de synthèse, Bobo-Dioulasso, PNGT - CILSS

Rivière D'arc Hélène 1991 "Du national au particulier : Le débat sur la décentralisation dans trois pays d'Amérique Latine (Brésil, Mexique, Cuba)" in : Cahiers des Amériques Latines $n^{\circ} 11, p p$. $-22$

Thomson James et Tall Mountaga, 1991 Décentralisation en matière de fourniture des services publics, gouvernance et gestion des ressources naturelles renouvelables au Mali contemporain, Club du Sahel - CILSS - Associates in Rural Development

Thomson J., Waldstein A., Gellar S., Miner J. 1989 Options pour promouvoir le contrôle et la gestion par les usagers des ressources naturelles renouvelables au Sahel, Associates in Rural Development

Thomson Jamie, 1992 Decentralization in Mali : action options, Bamako, USAID

\section{NOTES}

1.Le principe de la subsidiarité veut que les décisions soient prises par les institutions présentes à l'échelon spatial directement concerné. Il a pour objectifs une prise de décision rapide - car directement traitée par l'échelon concerné -, efficace - car considérée par des acteurs informés - et responsabilisant les institutions locales ou régionales.

2.Les politiques globales sont les politiques publiques qui dépassent le cadre sectoriel et posent, de manière implicite ou explicite, un choix de société.

3.Les acteurs cités sont, à mon avis, dans l'actuelle période d'équilibre institutionnel, les plus importants dans les processus d'élaboration des politiques globales du Mali. lis ont été sélectionnés car ils ont affirmés - pas toujours très clairement - des points de vue différents dans le cas particulier des modalités d'application de la politique de décentralisation. Par ailleurs, comme cette politique se trouve être normalement révélatrice de clivages idéologiques profonds, cette sélection devrait subsister à l'analyse d'autres politiques. Dernière remarque : il est intéressant de noter que deux des acteurs cités sont étrangers à la société malienne. Cela découle directement de la dépendance de l'Etat malien vis-à-vis des intervenants extérieurs et de la 
non-structuration des groupes sociaux maliens (il est par exemple clair que la même analyse faite au Burkina devrait tenir compte des syndicats de fonctionnaires et des organisations paysannes).

4.Chaque chapitre est synthétisé, sous forme de tableau, dans l'annexe 1 et une vue d'ensemble de la situation est offerte par le schéma de l'annexe 2. Par ailleurs, les projets de société de chacun des acteurs sont présentés par le tableau de l'annexe 3. 5.La société civile est définie ici en opposition, d'une part, à l'Etat et, d'autre part, aux communautés d'appartenance (ethnie, lignage, etc., considérées comme des structures non-démocratiques). Elle est donc composée de communautés d'adhésion - les associations, organisations, entreprises, partis politiques, syndicat, etc. - soit de toutes formes d'action collective organisée en dehors du champ des institutions étatiques et traditionnelles. Cette définition est, dans une large mesure, nuancée dans la conclusion de ce travail.

6.Dans l'ensemble des documents concernant l'actuelle politique de décentralisation, il est nulle part fait mention des anciennes institutions déconcentrées (comités et conseils locaux de développement) et du "fonds de développement local et régional" ! 7.Dans ce cas, il n'y aura pas démocratisation de la société malienne (donc constitution d'un Etat de droit), mais construction d'un Etat fonctionnant selon la logique du pouvoir coutumier (ce qui a toujours été le cas, tout au moins en partie, mais uniquement de manière implicite). $\mathrm{n}$ n'y a donc pas création d'un espace public ("espace qui se serait substitué à la société de type rural ou holiste du passé et capable d'agir parallèlement aux organisations corporatistes" (RIVIERE D'ARC, 1991: 13)), mais envahissement de l'espace public existant par les seules communautés d'appartenance. 8.Le Mali possède des régions productrices de coton ou d'autres biens d'exportation. Dans ces régions, les paysans ont des systèmes de production articulés au marché, donc dont la logique leur échappe partiellement. Cette articulation les "oblige à plus d'interdépendances, à plus de demande du secteur privé vis-à-vis du secteur public et donc confère une utilité aux politiques de décentralisation" (JACOB, 1993:3). n est donc probable que, dans ces régions, un intérêt certain existe pour les espaces de médiation mis à disposition des populations.

9.Il est possible qu'une partie des coûts induits par ces revendications soit pris en charge par la coopération internationale. En fait. la démultiplication des pouvoirs locaux offre aux ONG et aux collectivités locales des pays occidentaux des partenaires à leur taille.

10.Dans cette optique, la notion de service public est remplacée par celle de services payants réservés à ceux. qui ont accès au marché car, suivant la logique de la Banque Mondiale et du Fonds Monétaire International, ne vaut-il pas mieux des services qui existent pour les consommateurs solvables que des services publics qui n'existent pas? D'ailleurs, le discours de ces deux institutions concernant la société civile n'est pas écrit en terme de demande (la demande de services publics existe), mais en terme d'offre (c'est l'offre de services publics qui manque dans société sahélienne, c'est elle qui faut encourager au risque de dénaturer la forme du service, soit d'en faire un service privé); le rôle du médiateur revenant au marché.

11.Cette analyse demande a être nuancée. Le rôle donné par les institutions de Bretton Woods aux organisations locales et aux ONG relativise le recours unique au marché comme forme de régulation des relations entre acteurs.

12.Dans la pratique, la politique de "gestion des terroirs", pour être mobilisatrice, a besoin de s'appuyer sur des actions visibles et clairement identifiables par les 
villageois. Afin de répondre à cette obligation, les intervenants financent des micro-réalisations ayant pour objectif la production de biens publics. Ces actions permettent la mobilisation des populations et leur participation à des institutions locales. Par la suite, ces institutions, dont le fonctionnement est dans l'idéal organisé par les villageois, s'affirment comme les représentantes légitimes de la communauté villageoise et peuvent produire les règles d'utilisation des ressources naturelles. 13.Dans les discours du développement local, il y a souvent confusion entre services publics - offert à l'ensemble de la population -, services communautaires - offert aux membres d'une communauté d'adhésion - et services privés -offert à l'ensemble de la population contre une rémunération, dont ne bénéficient que les détenteurs du capital d'origine -. Jusqu'à présent, l'urgence des besoins a permis un amalgame de ces trois notions, mais la création, dans certaines régions, de véritables "structures administratives" parallèles à celle de l'Etat est en train modifier les données du débat 14.La possibilité de confits de compétence a clairement été évaluée par les acteurs à l'origine de la politique de décentralisation. Preuve en est l'essai de redéfinition des compétences de chacun des niveaux politico-administratifs (selon le principe de subsidiarité) et l'élaboration d'un mode de règlement des conflits prévisibles (Chauvie, $1993: 2-3)$.

15.Une vision synthétique du rôle imaginé pour chacun des éléments de la société malienne par les acteurs à l'origine de l'élaboration de la politique de décentralisation est produite, sous forme de tableau, à l'annexe 3.

16.La constitution et la structuration de la société civile ont donné lieu à de vifs débats lors des discussions sur les modalités concernant la mise en place de la politique de décentralisation. Les organisations d'aide au développement proposait une propagation de la politique de décentralisation répondant à la logique ascendante, donc organisée par étapes, régions par régions, et fonction des capacités de la société civile à investir les nouvelles institutions, alors que la classe politique tenait à imposer au même moment un cadre juridique contraignant sur l'ensemble du territoire national (et ce malgré les problèmes posés par une telle démarche, comme on l'a vu ci-dessus). Les institutions de Bretton Woods optent évidement pour cette seconde solution, puisqu'elles sont sûres que la société civile émergera spontanément, une fois réunies les conditions permettant l'apparition des lois du marché.

\section{AUTEUR}

\section{JULIEN FÉLIX}

Institut Universitaire d'Etudes du Développement (Genève). 